\title{
Téoros
}

Revue de recherche en tourisme

\section{Renseignements touristiques et... nouvelles technologies}

\section{Pierre Duguay}

Volume 8, numéro 1, mars 1989

France-Québec

URI : https://id.erudit.org/iderudit/1080357ar

DOI : https://doi.org/10.7202/1080357ar

Aller au sommaire du numéro

Éditeur(s)

Université du Québec à Montréal

ISSN

0712-8657 (imprimé)

1923-2705 (numérique)

Découvrir la revue

Citer cet article

Duguay, P. (1989). Renseignements touristiques et... nouvelles technologies.

Téoros, 8(1), 32-32. https://doi.org/10.7202/1080357ar d'utilisation que vous pouvez consulter en ligne.

https://apropos.erudit.org/fr/usagers/politique-dutilisation/ 


\section{Renseignements touristiques et...

La guerre des boutons $\mathrm{n}^{+}$aura pas lieu de sitôt. S'il est un domaine où le "médium" n'est pas encore le "message" - et ne le sera peut-être jamais - c'est bien le secteur du renseignement touristique. À quoi cela tient-il? A beaucoup de facteurs reliés autant aux contenus emmagasinés et vêhiculés qu'aux supports de transmission.

Le touriste est avant tout un voyeur et un tactile. Je ne pense pas que l' 6 cran noir de son Minitel - ou de son Alex - truffé de petits points blancs linéaires puisse constituer véritablement une invitation au voyage. Et pourtant, il s'agit là de la technologie proclamée la plus avancée en la matière! Alors, les autres...

\section{Les technologies}

Les autres "nouvelles technologies", ce sont d'abord les "lignes téléphoniques". Elles ont l'avantage d'être bi-directionnelles, de porter la voix humaine et de provoquer ainsi l'ajustement nécessaire entre les demandes d'information précise, circonstanciée, et les réponses données de façon adaptée.

Ici, les contenus sont obligatoirement traités en fonction des besoins exprimés par le client et se trouvent d'entrée de jeu à s'inscrire dans une approche marketing qui force - par la rétroaction - l'ajustement des données et mème la promotion des produits touristiques. Compte tenu du nombre et de la diversité des appels, la technologie "lignes teléphoniques" nécessite un support informatique important pour le traitement rétroactif des informations demandées et la réinjection, dans les banques de données actuellement manuelles, des renseignements mis à jour.

A mon avis, il s'agit la de la technologic qui a le plus d'avenir puisqu'elle abolit la distance entre le touriste actuel et potentiel et son point de contact. Elle peut aussi répondre à un nombre et à un genre presque illimités de demandes chaque jour. Lorsque la téléphonie ajoutera la dimension visuelle et qu'en plus des visages, l'écran pourra contenir des images de lieux, d'attraits, d'événements, de parcours, alors là, il y aura non seulement la guerre des boutons, à savoir qu'elle est la meilleure technologie, mais aussi la guerre tout court, c'est-à-dire la compétition entre les services de renseignements des différents états ou régions.

\footnotetext{
" Pierre Duguay est coordonnateur du projet Infotouriste
} de Montréal
L'autre "nouvelle technologie", qui est d'ailleurs apparentée à la première, consiste à l'utilisation du véhicule "télévision par câble". Sur une chaîne donnée l'information touristique peut être accessible et des produits - notamment des billets de spectacles - sont disponibles. Il s'agit d'appeler. Cette technologic se permettra bientôt dêtre bidirectionnelle mais ce sera un dialogue de machines. L'avantage consiste au fait que l'information est jumelée à une possible "transaction", à savoir un achat de produit touristique. On parle de billets de spectacles; ce pourrait être un forfait séjour, une réservation d'hôtel ou de transport. L'information cependant est nécessairement "en boilte" et pas trop ajustée aux besoins du client.

Dans cette panoplie de supports technologiques, il existe également des "bidules". $\mathrm{Ce}$ sont des consoles, apparentées à des terminaux d'ordinateurs, qui sont programmées pour diffuser une information sur une région, ses attraits, ses produits, ses événements ou encore sur des hótels ou des restaurants. II s'agit d'accéder à la table des matières et ensuite de choisir la rubrique souhaitée. Ces bidules doivent être consultés sur place, c'est-à-dire dans un centre touristique, un hall d'hôtel, une salle de congrès. Leur utilisation est donc réduite à une personne à la fois et leur contenu est limité.

\section{Le contenu}

Depuis le début, on se réferre régulièrement au contenu de "l'information touristique" . Il y a en effet une certaine distorsion entre le traitement des données et la capacité des divers supports technologiques. Cela tient au fait que la constitution des banques de renseignements s'est effectuée à partir de catalogues, de guides issus directement de la "galaxie Gutenberg", donc d'un processus linéaire, séquentiel, descriptif et neutre. Lorsque ce contenu doit subir le jeu de l'interaction et de l'ajustement aux questions des touristes, son efficacité ne dépasse pas celle des guides écrits ou des bottins téléphoniques. Un développement important du traitement des données reste donc à faire pour que les renseignements acquièrent le niveau de souplesse et d'adaptation requis. Dans cette même perspective, les contenus devraient être davantage partie intégrante des stratégies de marketing et de la recherche des besoins des clients. Ils devraient également déboucher sur des transactions possibles et immédiates en matière de réservation et de ventes de certains produits touristiques de base (hôtels, transport, spectacles).

\section{Une expérience multi-média: Infotouriste}

Au printemps 1989, on ouvrira à Montréal un centre intégré d'accueil et de renseignements touristiques. Intêgré, puisque l'information touristique sur Montréal, les régions du Québec et du Canada seront disponibles au même endroit (en plus de trente-deux lignes téléphoniques accessibles à partir de presque toute l'Amérique du Nord), intégré puisque cette information se situera dans un environnement promotionnel sophistiqué (mur vidéo, tableaux électroniques, murales, vitrines thématiques), intégré aussi, puisque, au même endroit, le touriste pourra faire tous ses achats de services touristiques: tours de ville ou de régions, forfaits sćjour à Montréal ou en province, spectacles, événements, etc.

Ce caractère intégré se répercutera également dans l'utilisation des diverses technologies évoquées plus haut: lignes téléphoniques, Minitel ou Alex, "bidules", billetteries automatiques, mais dans le contexte d'un marketing centré le plus possible sur les besoins du client, besoins de renseignements mais également de conseils et d'achats.

Cette expérience est à suivre et à examiner attentivement. Mais on peut d'ores et déja prévoir que le renseignement touristique et ses supports technologiques subiront des ajustements importants en fonction des demandes exprimées. El surtout que, s'il est vrai que le "médium est le message" , rien ne remplacera jamais une communication humaine chaleureuse et efficace, au comptoir ou au téléphone, entre un préposé et un visiteur.

\section{Conclusion}

Voir, toucher, sentir un ailleurs. Se découvrir soi-même semblable et différent dans un endroit autre. Il reste beaucoup à faire pour que les "technologies nouvelles" puissent servir de support efficace à cette trame de fond et présenter cet "autre". II reste surtout à approfondir les besoins du touriste, sa neutralité, ses attentes, son identité. Après quoi, les immenses possibilités de ces instruments et leur contenu pourront être orientées vers des programmations de plus en plus personnalisces et capables d'abolir toutes les distances. 